\title{
ON PROPER HELICES AND EXTRINSIC SPHERES IN PSEUDO-RIEMANNIAN GEOMETRY
}

\author{
By
}

\author{
Hwa Hon Song, Takahisa KimURA and Naoyuki KoIKe
}

\begin{abstract}
In this paper, we define the notion of a proper helix of order $d$ in a pseudo-Riemannian manifold and investigate those curves in a totally umbilical pseudo-Riemannian submanifold.
\end{abstract}

\section{Introduction.}

In Riemannian geometry, properties of regular curves are well discribed by the Frenet formula. In [8], K. Sakamoto called a regular curve which has constant curvatures of osculating order $d$ a helix of order $d$. Note that a helix of order one (resp. two) is a geodesic (resp. circle). The research of geodesics, circles and helices (of order three) in Riemannian submanifold theory, has been done by K. Nomizu and K. Yano ([5]), H. Nakagawa ([2]), K. Sakamoto ([7]) and other geometricians. Furthermore, K. Sakamoto also has investigated helices of general order in the theory (cf. [8]). For regular curves in a pseudoRiemannian manifold, we can not necessarily define a formula corresponding to the Frenet formula. Especially, we call a regular curve with a formula corresponding to the Frenet formula a proper curve. Furthermore, we call a proper curve which has constant curvatures of osculating order $d$ a proper helix of order $d$. N. Abe, Y. Nakanishi and S. Yamaguchi defined general circles and helices (of order three) in a pseudo-Riemannian manifold. They investigated those curves in a pseudo-Riemannian submanifold (cf. [1], [3], [4]). We shall investigate proper helices of general order in a totally umbilical pseudoRiemannian submanifold.

The authors would like to express his hearty thanks to Professor S. Yamaguchi for his constant encouragement and various advice. They also wish to express sincere gratitude to thank Professor N. Abe for his valuable suggestions.

Received June 16, 1994 


\section{§1. Notations and Basic Equations.}

In this paper, the differentiability of all geometric objects will be $C^{\infty}$. Let $M$ be a pseudo-Riemannian submanifold in pseudo-Riemannian manifold $\bar{M}$ isometrically immersed by $f$ and denote by $g$ (resp. $\bar{g}$ ) the pseudo-Riemannian metric of $M$ (resp. $\bar{M}$ ). For all local formulas and calculations, we may assume $f$ as an imbedding and thus we shall often identify $p \in M$ with $f(p) \in \bar{M}$. The tangent space $T_{p} M$ at $p$ is identified with a subspace $f_{*}\left(T_{p} M\right)$ of the tangent space $T_{p} \bar{M}$. We put $\|X\|:=\sqrt{|\bar{g}(X, X)|}$ for $X \in T_{p} \bar{M}$. We denote the tangent bundle of $M$ by $T M$ and the normal bundle by $T^{\perp} M$. Let $\bar{\nabla}$ and $\nabla$ be the Levi-Civita connections of $\bar{M}$ and $M$, respectively. Then the Gauss formula is given by

$$
\bar{\nabla}_{X} Y=\nabla_{X} Y+B(X, Y) \text {, }
$$

where $X$ and $Y$ are tangent vector fields of $M$ and $B$ is the second fundamental form of $M$. The Weingarten formula is given by

$$
\bar{\nabla}_{x} \xi=-A_{\xi} X+\nabla_{x}^{\perp} \xi
$$

where $X$ (resp. $\xi$ ) is a tangent (resp. normal) vector field of $M$ and $A$ (resp. $\nabla^{\perp}$ ) is the shape operator (resp. the normal connection) of $M$. Clearly $A$ is related to $B$ as

$$
g\left(A_{\xi} X, Y\right)=\bar{g}(B(X, Y), \xi)
$$

The mean curvature vector field $H$ of $M$ is defined by

$$
H:=\frac{1}{n} \sum_{i=1}^{n} g\left(e_{i}, e_{i}\right) B\left(e_{i}, e_{i}\right)
$$

where $n=\operatorname{dim} M$ and $\left\{e_{1}, \cdots, e_{n}\right\}$ is an orthonormal frame of $M$. If the second fundamental form $B$ satisfies

$$
B(X, Y)=g(X, Y) H
$$

for every tangent vector fields $X, Y$ of $M$, then $M$ is called a totally umbilical submanifold. The mean curvature vector field $H$ is said to be parallel if $\nabla_{X}^{\perp} H=0$ for every tangent vector field $X$ of $M$. A totally umbilical submanifold with the parallel mean curvature vector field is called an extrinsic sphere. If the second fundamental form $B$ vanishes identically, then $M$ is called a totally geodesic submanifold of $\bar{M}$.

Next we shall define the notion of a proper helix of order $d$ in a pseudoRiemannian manifold $N$. Let $\sigma: I \rightarrow N$ be a non-null curve in $N$ parametrized by the arclength $s$, where $I$ is an open interval of the real line $R$. We denote the 
tangent vector field of $\sigma$ by $v_{0}$. We assume that $\sigma$ satisfies the following Frenet formula:

$$
\left\{\begin{array}{c}
\nabla_{v_{0}} v_{0}=\lambda_{1} v_{1} \\
\nabla_{v_{0}} v_{1}+\varepsilon_{0} \varepsilon_{1} \lambda_{1} v_{0}=\lambda_{2} v_{2} \\
\nabla_{v_{0}} v_{2}+\varepsilon_{1} \varepsilon_{2} \lambda_{2} v_{1}=\lambda_{3} v_{3} \\
\vdots \\
\nabla_{v_{0}} v_{d-2}+\varepsilon_{d-3} \varepsilon_{d-2} \lambda_{d-2} v_{d-3}=\lambda_{d-1} v_{d-1} \\
\nabla_{v_{0}} v_{d-1}+\varepsilon_{d-2} \varepsilon_{d-1} \lambda_{d-1} v_{d-2}=0
\end{array}\right.
$$

where

$$
\left\{\begin{array}{l}
\lambda_{1}:=\left\|\nabla_{v_{0}} v_{0}\right\|>0 \\
\lambda_{i}:=\left\|\nabla_{v_{0}} v_{i-1}+\varepsilon_{i-2} \varepsilon_{i-1} \lambda_{i-1} v_{i-2}\right\|>0, \quad(2 \leq i \leq d-1) \\
\varepsilon_{j}:=g\left(v_{j}, v_{j}\right)(= \pm 1) \quad(0 \leq j \leq d-1) \quad \text { on } I .
\end{array}\right.
$$

We call such a curve a proper curve of order $d, \lambda_{i}$ the $i$-th curvature and $v_{0}, \ldots, v_{d-1}$ the Frenet frame field. Furthermore, if $\lambda_{i}(1 \leq i \leq d-1)$ are constant along $\sigma$, then we call this curve a proper helix of order $d$.

\section{§2. Proper helices in a totally umbilical pseudo-Riemannian submanifold.}

Let $M$ be a totally umbilical pseudo-Riemannian submanifold in a pseudoRiemannian manifold $\bar{M}$ isometrically immersed by $f$ and $\sigma$ a proper helix of order $d$ in $M$. We denote a curve $f \circ \sigma$ in $\bar{M}$ by $\bar{\sigma}$. Assume that $\bar{\sigma}$ is a proper helix of order $\bar{d}$. Let $\lambda_{1}, \cdots, \lambda_{d-1}$ (resp. $\bar{\lambda}_{1}, \cdots, \bar{\lambda}_{\bar{d}_{-1}}$ ) be the curvatures of $\sigma$ (resp. $\bar{\sigma})$ and $v_{0}, \cdots, v_{d-1}\left(\right.$ resp. $\bar{v}_{0}, \cdots, \bar{v}_{\bar{d}-1}$ ) the Frenet frame field of $\sigma$ (resp. $\bar{\sigma}$ ). For convenience, let $\lambda_{i}=0, v_{i}=0, \bar{\lambda}_{j}=0$ and $\bar{v}_{j}=0(i \geq d, j \geq \bar{d})$. Set $\varepsilon_{i}:=g\left(v_{i}, v_{i}\right)$ and $\bar{\varepsilon}_{i}:=\bar{g}\left(\bar{v}_{i}, \bar{v}_{i}\right)(i \geq 0)$. We define $\nabla_{v_{0}}^{\perp(i)} H(i \geq 0)$ by $\nabla_{v_{0}}^{\perp(0)} H:=H$ and $\nabla_{v_{0}}^{\perp(i)} H:=\nabla_{v_{0}}^{\perp}$ $\left(\nabla_{v_{0}}^{\perp}{ }^{(i-1)} H\right)(i \geq 1)$. Also, we define $\beta_{i, j}$ and $\bar{\beta}_{i, j}(i \geq j \geq 1, i+j:$ even $)$ by

$$
\left\{\begin{array}{l}
\beta_{1,1}=\lambda_{1}, \quad \bar{\beta}_{1,1}=\bar{\lambda}_{1} \\
\beta_{i, i}=\lambda_{i} \beta_{i-1, i-1}, \quad \bar{\beta}_{i, i}=\bar{\lambda}_{i} \bar{\beta}_{i-1, i-1} \quad(i \geq 2) \\
\beta_{2 i+1,1}=-\varepsilon_{1} \varepsilon_{2} \lambda_{2} \beta_{2 i, 2}, \quad \bar{\beta}_{2 i+1,1}=-\bar{\varepsilon}_{1} \bar{\varepsilon}_{2} \bar{\lambda}_{2} \bar{\beta}_{2 i, 2} \quad(i \geq 1) \\
\beta_{i, j}=-\varepsilon_{j} \varepsilon_{j+1} \lambda_{j+1} \beta_{i-1, j+1}+\lambda_{j} \beta_{i-1, j-1} \quad(i>j \geq 2) \\
\bar{\beta}_{i, j}=-\bar{\varepsilon}_{j} \bar{\varepsilon}_{j+1} \bar{\lambda}_{j+1} \bar{\beta}_{i-1, j+1}+\bar{\lambda}_{j} \bar{\beta}_{i-1, j-1} \quad(i>j \geq 2) .
\end{array}\right.
$$


LEMMA 2.1. The vector fields $v_{i}(i \geq 0)$ and $\bar{v}_{j}(j \geq 0)$ along $\sigma$ are related as follows:

$\left(F_{0}\right) \quad \bar{v}_{0}=v_{0}$,

$\left(F_{2 i-1}\right) \quad \sum_{j=1}^{i} \bar{\beta}_{2 i-1,2 j-1} \bar{v}_{2 j-1}=\sum_{j=1}^{i} \beta_{2 i-1,2 j-1} v_{2 j-1}+\varepsilon_{0} \nabla_{v_{0}}^{\perp(2 i-2)} H \quad(i \geq 1)$,

$\left(F_{2 i}\right) \quad \sum_{j=1}^{i} \bar{\beta}_{2 i, 2 j} \bar{v}_{2 j}=\sum_{j=1}^{i} \beta_{2 i, 2 j} v_{2 j}+\varepsilon_{0} \nabla_{v_{0}}^{\perp(2 i-1)} H \quad(i \geq 1)$.

Proof. By using (1.1), the Frenet formulas and the assumption that $M$ is totally umbilic, we get

$$
\bar{\lambda}_{1} \bar{v}_{1}=\bar{\nabla}_{v_{0}} v_{0}=\nabla_{v_{0}} v_{0}+\varepsilon_{0} H=\lambda_{1} v_{1}+\varepsilon_{0} H .
$$

Thus we obtain $\left(F_{1}\right)$. Operating $\bar{\nabla}_{v_{0}}$ to $\left(F_{1}\right)$, we get

$$
\bar{\beta}_{1,1}\left(-\varepsilon_{0} \bar{\varepsilon}_{1} \bar{\lambda}_{1} v_{0}+\bar{\lambda}_{2} \bar{v}_{2}\right)=\beta_{1,1}\left(-\varepsilon_{0} \varepsilon_{1} \lambda_{1} v_{0}+\lambda_{2} \bar{v}_{2}\right)-\varepsilon_{0} \bar{g}(H, H) v_{0}+\varepsilon_{0} \nabla_{v_{0}}^{\perp} H,
$$

where we use (1.1), (1.2), the Frenet formulas and the assumption that $M$ is totally umbilic. By noticing $\left\{v_{0}\right\}^{\perp}$-component of this equality, we see that

$$
\bar{\lambda}_{2} \bar{\beta}_{1,1} \bar{v}_{2}=\lambda_{2} \beta_{1,1} v_{2}+\varepsilon_{0} \nabla_{v_{0}}^{\perp} H,
$$

which implies $\left(F_{2}\right)$ by $(2.1)$. Assume that $\left(F_{2 k}\right)$ holds. Operating $\bar{\nabla}_{v_{0}}$ to $\left(F_{2 k}\right)$, we have

$$
\begin{aligned}
\sum_{j=1}^{k} \bar{\beta}_{2 k, 2 j} \bar{\nabla}_{v_{0}} \bar{v}_{2 j}=\sum_{j=1}^{k} \beta_{2 k, 2 j} \nabla_{v_{0}} v_{2 j} & -\varepsilon_{0} \bar{g}\left(\nabla_{v_{0}}^{\perp}{ }^{(2 k-1)} H, H\right) v_{0} \\
& +\varepsilon_{0} \nabla_{v_{0}}^{\perp(2 k)} H,
\end{aligned}
$$

where we use (1.1), (1.2) and the assumption that $M$ is totally umbilic. Furthermore, by using the Frenet formulas and (2.1), we have

$$
\sum_{j=1}^{k+1} \bar{\beta}_{2 k+1,2 j-1} \bar{v}_{2 j-1}=\sum_{j=1}^{k+1} \bar{\beta}_{2 k+1,2 j-1} v_{2 j-1}-\varepsilon_{0} \bar{g}\left(\nabla_{v_{0}}^{\perp(2 k-1)} H, H\right) v_{0}+\varepsilon_{0} \nabla_{v_{0}}^{\perp(2 k)} H
$$

Therefore, by noticing Span $\left\{v_{0}\right\}^{\perp}$-component of this equality, we obtain $\left(F_{2 k+1}\right)$. Similarly, by operating $\bar{\nabla}_{v_{0}}$ to $\left(F_{2 k+1}\right)$ and using the Frenet formulas and (2.1), we also have

$$
\begin{aligned}
-\varepsilon_{0} \bar{\varepsilon}_{1} \bar{\lambda}_{1} \bar{\beta}_{2 k+1,1} v_{0}+\sum_{j=1}^{k+1} \bar{\beta}_{2 k+2,2 j} \bar{v}_{2 j} & =-\varepsilon_{0}\left\{\varepsilon_{1} \lambda_{1} \beta_{2 k+1,1}+\bar{g}\left(\nabla_{v_{0}}^{\perp(2 k)} H, H\right)\right\} v_{0} \\
& +\sum_{j=1}^{k+1} \beta_{2 k+2,2 j} v_{2 j}+\nabla_{v_{0}}^{\perp(2 k+1)} H .
\end{aligned}
$$


Thus, by noticing $\operatorname{Span}\left\{v_{0}\right\}^{\perp}$-component of this equality, we also have $\left(F_{2 k+2}\right)$. Therefore, by the induction, we see that $\left(F_{i}\right)$ holds for every $i \geq 0$.

Now we define column vectors $\boldsymbol{b}_{i}(i \geq 3)$ and matrices $\boldsymbol{B}_{i}(i \geq 1)$ by

$$
\boldsymbol{b}_{2 j-1}:=\left(\begin{array}{c}
\beta_{2 j-1,1} \\
\beta_{2 j-1,3} \\
\vdots \\
\beta_{2 j-1,2 j-3}
\end{array}\right), \quad \boldsymbol{b}_{2 j}:=\left(\begin{array}{c}
\beta_{2 j, 2} \\
\beta_{2 j, 4} \\
\vdots \\
\beta_{2 j, 2 j-2}
\end{array}\right) \quad(j \geq 2)
$$

and

$$
\begin{aligned}
\boldsymbol{B}_{2 j-1}: & =\left(\begin{array}{cccc}
\beta_{1,1} & 0 & \cdots & 0 \\
\beta_{3,1} & \beta_{3,3} & \ddots & \vdots \\
\vdots & \vdots & \ddots & 0 \\
\beta_{2 j-1,1} & \beta_{2 j-1,3} & \cdots & \beta_{2 j-1,2 j-1}
\end{array}\right), \\
\boldsymbol{B}_{2 j}: & =\left(\begin{array}{cccc}
\beta_{2,2} & 0 & \cdots & 0 \\
\beta_{4,2} & \beta_{4,4} & \ddots & \vdots \\
\vdots & \vdots & \ddots & 0 \\
\beta_{2 j, 2} & \beta_{2 j, 4} & \cdots & \beta_{2 j, 2 j}
\end{array}\right) \quad(j \geq 1) .
\end{aligned}
$$

Also, we define formal column vectors $\boldsymbol{V}_{i}(i \geq 1)$ and $\boldsymbol{H}_{i}(i \geq 0)$ whose components are vector fields along $\sigma$ by

$$
\boldsymbol{V}_{2 j-1}:=\left(\begin{array}{c}
v_{1} \\
v_{3} \\
\vdots \\
v_{2 j-1}
\end{array}\right), \boldsymbol{V}_{2 j}:=\left(\begin{array}{c}
v_{2} \\
v_{4} \\
\vdots \\
v_{2 j}
\end{array}\right) \quad(j \geq 1)
$$

and

$$
\boldsymbol{H}_{2 j}:=\left(\begin{array}{c}
H \\
\nabla_{v_{0}}^{\perp(2)} H \\
\vdots \\
\nabla_{v_{0}}^{\perp(2 j)} H
\end{array}\right), \boldsymbol{H}_{2 j+1}:=\left(\begin{array}{c}
\nabla_{v_{0}}^{\perp} H \\
\nabla_{v_{0}}^{\perp(3)} H \\
\vdots \\
\nabla_{v_{0}}^{\perp}{ }^{(2 j+1)} H
\end{array}\right) \quad(j \geq 0) .
$$

Similarly, we define $\overline{\boldsymbol{b}}_{i}(i \geq 3), \overline{\boldsymbol{B}}_{i}(i \geq 1)$ and $\overline{\boldsymbol{V}}_{i}(i \geq 1)$ in terms of $\overline{\boldsymbol{\beta}}_{i, j}$ and $\bar{v}_{i}$ instead of $\beta_{i, j}$ and $v_{i}$. Note that $\boldsymbol{B}_{i}(i \leq d-1) \overline{\boldsymbol{B}}_{i}(i \leq \bar{d}-1)$ are nonsingular by (2.1). By using these notations, $\left(F_{i}\right)$ is expresses as follows: 


$$
{ }^{\prime} \overline{\boldsymbol{b}}_{i} \overline{\boldsymbol{V}}_{\mathrm{i}-2}+\bar{\beta}_{i, i} \bar{v}_{i}={ }^{\prime} \boldsymbol{b}_{i} \boldsymbol{V}_{\mathrm{i}-2}+\beta_{i, i} v_{i}+\varepsilon_{0} \nabla_{\boldsymbol{v}_{0}}^{\perp(i-1)} H .(i \geq 3)
$$

Moreover, the systems $\left(F_{1}\right),\left(F_{3}\right), \cdots,\left(F_{2 i-1}\right)(i \geq 1)$ and $\left(F_{2}\right),\left(F_{4}\right), \cdots,\left(F_{2 i}\right)(i \geq 1)$ are expressed as

$$
\begin{aligned}
& \bar{B}_{2 i-1} \bar{V}_{2 i-1}=B_{2 i-1} V_{2 i-1}+\varepsilon_{0} H_{2 i-2}, \\
& \bar{B}_{2 i} \bar{V}_{2 i}=B_{2 i} V_{2 i}+\varepsilon_{0} H_{2 i-1},
\end{aligned}
$$

respectively. Thus we have

$$
\bar{B}_{i} \bar{V}_{i}=B_{i} V_{i}+\varepsilon_{0} H_{i-1}, \quad(i \geq 1) .
$$

From (2.2) and (2.3), we have

$$
\begin{aligned}
\left(M F_{i}\right)-\overline{\boldsymbol{\beta}}_{i, i} \bar{v}_{i}+\boldsymbol{\beta}_{i, i} v_{i}= & \left({ }^{t} \overline{\boldsymbol{b}}_{i} \overline{\boldsymbol{B}}_{i-2}^{-1} \boldsymbol{B}_{i-2}-{ }^{t} \boldsymbol{b}_{i}\right) \boldsymbol{V}_{i-2} \\
& +\varepsilon_{0}\left({ }^{t} \overline{\boldsymbol{b}}_{i} \overline{\boldsymbol{B}}_{i-2}^{-1} \boldsymbol{H}_{i-3}-\nabla_{\boldsymbol{v}_{0}}^{\perp(i-1)} H\right) \quad(3 \leq i \leq \bar{d}+1) .
\end{aligned}
$$

LEMMA 2.2. The inequality $d \leq \bar{d} \leq d+r$ holds, where $r$ is the codimension of $M$ in $\overline{\boldsymbol{M}}$.

Proof. Suppose $d>\bar{d}$. Then we have $v_{\bar{d}} \neq 0$ and $\bar{v}_{\bar{d}}=0$. Hence, it follows from $\left(M F_{\bar{d}}\right)$ that

$$
\begin{aligned}
\boldsymbol{\beta}_{\bar{d}, \bar{d}} v_{\bar{d}}=\left({ }^{\prime} \overline{\boldsymbol{b}}_{\bar{d}} \overline{\boldsymbol{B}}_{\bar{d}-2}^{-1} \boldsymbol{B}_{\bar{d}-2}-{ }^{t} \boldsymbol{b}_{\bar{d}}\right) \boldsymbol{V}_{\bar{d}-2} \\
+\varepsilon_{0}\left({ }^{\prime} \overline{\boldsymbol{b}}_{\bar{d}} \overline{\boldsymbol{B}}_{\bar{d}-2}^{-1} \boldsymbol{H}_{\bar{d}-3}-\nabla_{v_{0}}^{\perp(\bar{d}-1)} H\right) .
\end{aligned}
$$

Since $v_{\bar{d}}$ is linearly independent of $v_{i}(i \leq \bar{d}-2)$ and $\nabla_{v_{0}}^{\perp}{ }^{(i)} H(i \leq \bar{d}-1)$, we have $\beta_{\bar{d}, \bar{d}} v_{\bar{d}}=0$. From (2.1) and $d>\bar{d}, \beta_{\bar{d}, \bar{d}}=\lambda_{1} \lambda_{2} \cdots \lambda_{\bar{d}} \neq 0$ is deduced. Therefore, we have $v_{\bar{d}}=0$. This contradicts $d>\bar{d}$. Thus we have $d \leqq \bar{d}$. The remaining part is trivial.

LEMMA 2.3. (i) If $\bar{d}=d(\geq 3)$, then $\nabla_{\boldsymbol{v}_{0}}^{\perp(d-1)} H={ }^{\prime} \boldsymbol{b}_{d} \boldsymbol{B}_{d-2}{ }^{-1} \boldsymbol{H}_{d-3}$ holds.

(ii) If $\bar{d}=d+1(d \geq 2)$, then $\nabla_{v_{0}}^{\perp(d)} H={ }^{\prime} \boldsymbol{b}_{d+1} \boldsymbol{B}_{d-1}{ }^{-1} \boldsymbol{H}_{d-2}$ holds.

Proof. (i) By the assumption, $v_{d}=0$ and $\bar{v}_{d}=0$ holds. Substituting these to $\left(M F_{d}\right)$, we have

$$
\left({ }^{\prime} \overline{\boldsymbol{b}}_{d} \overline{\boldsymbol{B}}_{d-2}^{-1} \boldsymbol{B}_{d-2}-{ }^{\prime} \boldsymbol{b}_{d}\right) \boldsymbol{V}_{d-2}+\varepsilon_{0}\left({ }^{\prime} \overline{\boldsymbol{b}}_{d} \overline{\boldsymbol{B}}_{d-2}^{-1} \boldsymbol{H}_{d-3}-\nabla_{\boldsymbol{v}_{0}}^{+(d-1)} H\right)=0 .
$$

By noticing the tangential component and the normal component of this equality, we have 


$$
{ }^{t} \overline{\boldsymbol{b}}_{d} \overline{\boldsymbol{B}}_{d-2}^{-1}={ }^{t} \boldsymbol{b}_{d} \boldsymbol{B}_{d-2}{ }^{-1}
$$

and

$$
\nabla_{\boldsymbol{v}_{0}}^{\perp(d-1)} H={ }^{\prime} \overline{\boldsymbol{b}}_{d} \overline{\boldsymbol{B}}_{d-2}^{-1} \boldsymbol{H}_{d-3}
$$

These imply

$$
\nabla_{v_{0}}^{\perp(d-1)} H={ }^{t} \boldsymbol{b}_{d} \boldsymbol{B}_{d-2}{ }^{-1} \boldsymbol{H}_{d-3} .
$$

(ii) By the assumption, $v_{d+1}=0$ and $\bar{v}_{d+1}=0$ holds. Substituting these to $\left(M F_{d+1}\right)$, we have

$$
\left({ }^{t} \overline{\boldsymbol{b}}_{d+1} \overline{\boldsymbol{B}}_{d-1}^{-1} \boldsymbol{B}_{d-1}-{ }^{t} \boldsymbol{b}_{d+1}\right) \boldsymbol{V}_{d-1}+\varepsilon_{0}\left({ }^{t} \overline{\boldsymbol{b}}_{d+1} \overline{\boldsymbol{B}}_{d-1}^{-1} \boldsymbol{H}_{d-2}-\nabla_{\boldsymbol{v}_{0}}^{+(d)} H\right)=0 .
$$

By noticing the tangential component and the normal component of this equality, we have

$$
{ }^{t} \overline{\boldsymbol{b}}_{d+1} \overline{\boldsymbol{B}}_{d-1}^{-1}={ }^{t} \boldsymbol{b}_{d+1} \boldsymbol{B}_{d-1}{ }^{-1}
$$

and

$$
\nabla_{v_{0}}^{\perp(d)} H={ }^{t} \overline{\boldsymbol{b}}_{d+1} \overline{\boldsymbol{B}}_{d-1}^{-1} \boldsymbol{H}_{d-2}
$$

These imply

$$
\nabla_{\boldsymbol{v}_{0}}^{\perp(d)} H={ }^{t} \boldsymbol{b}_{d+1} \boldsymbol{B}_{d-1}^{-1} \boldsymbol{H}_{d-2} .
$$

Since ${ }^{t} \boldsymbol{b}_{2 i+1} \boldsymbol{B}_{2 i-1}{ }^{-1}(1 \leq 2 i-1 \leq d-1)$ is the solution of the equation $\left(x_{1}, \cdots\right.$, $\left.x_{i}\right) \boldsymbol{B}_{2 i-1}={ }^{t} \boldsymbol{b}_{2 i+1}$, by Cramér formula, we have

$$
\begin{array}{r}
{ }^{t} \boldsymbol{b}_{2 i+1} \boldsymbol{B}_{2 i-1}{ }^{-1}=\frac{1}{\left|\boldsymbol{B}_{2 i-1}\right|}\left(P_{2 i+1,1}\left(\lambda_{1}, \cdots, \lambda_{2 i}\right), \cdots\right. \\
\left.\cdots, P_{2 i+1, i}\left(\lambda_{1}, \cdots, \lambda_{2 i}\right)\right),
\end{array}
$$

where $P_{2 i+1, j}\left(\lambda_{1}, \cdots, \lambda_{2 i}\right)(1 \leq j \leq i)$ is the determinant replaced the $j$-th row of $\left|\boldsymbol{B}_{2 i-1}\right|$ by ${ }^{t} b_{2 i+1}$. Similarly, we have

$$
\begin{aligned}
&{ }^{t} \boldsymbol{b}_{2 i} \boldsymbol{B}_{2 i-2}{ }^{-1}=\frac{1}{\left|\boldsymbol{B}_{2 i-2}\right|}\left(P_{2 i, 1}\left(\lambda_{1}, \cdots, \lambda_{2 i-1}\right), \cdots\right. \\
&\left.\cdots, P_{2 i, i-1}\left(\lambda_{1}, \cdots, \lambda_{2 i-1}\right)\right) \\
& \\
&(2 \leq 2 i-2 \leq d-1),
\end{aligned}
$$

where $P_{2 i, j}\left(\lambda_{1}, \cdots, \lambda_{2 i-1}\right)(1 \leq j \leq i-1)$ is the determinant replaced the $j$-th row of $\left|\boldsymbol{B}_{2 i-2}\right|$ by ${ }^{t} \boldsymbol{b}_{2 i}$. Then we have the following lemma.

LEMMA 2.4. (i) The polynomial $P_{2 i+1, j}\left(\lambda_{1}, \cdots, \lambda_{2 i}\right)(1 \leq j \leq i)$ is a homogeneous 
polynomial of degree $\left(i^{2}+2 i-2 j+2\right)$ and $P_{2 i, j}\left(\lambda_{1}, \cdots, \lambda_{2 i-1}\right)(1 \leq j \leq i-1)$. is a homogeneous polynomial of degree $\left(i^{2}+i-2 j\right)$.

(ii) The polynomial $P_{2 i+1,1}\left(\lambda_{1}, \cdots, \lambda_{2 i}\right)$ is expressed as follows:

$$
P_{2 i+1,1}\left(\lambda_{1}, \cdots, \lambda_{2 i}\right)=-\varepsilon_{1} \varepsilon_{2} \cdots \varepsilon_{2 i} \lambda_{2} \lambda_{4} \cdots \lambda_{2 i}\left|B_{2 i}\right|
$$

ProOF. (i) By (2.1), we see that $\beta_{i, j}$ is a homogeneous polynomial of degree $i$ with variables $\lambda_{1}, \cdots, \lambda_{i}$. Hence the conclusion is directly deduced from the definitions of $P_{2 i+1, j}\left(\lambda_{1}, \cdots, \lambda_{2 i}\right)$ and $P_{2 i, j}\left(\lambda_{1}, \cdots, \lambda_{2 i-1}\right)$.

(ii) Define $\hat{\beta}_{j, k}(j>k \geq 1, j+k$ : even) by

$$
\hat{\beta}_{j, k}= \begin{cases}0 & (j>k=1) \\ \lambda_{k} \beta_{j-1, k-1} & (j>k>1) .\end{cases}
$$

Then, from (2.1), we have

$$
\left(b_{j, k}\right) \quad \beta_{j, k}=-\varepsilon_{k} \varepsilon_{k+1} \lambda_{k+1} \beta_{j-1, k+1}+\hat{\beta}_{j, k} \quad(j>k>2) .
$$

Also, we define a matrix $C_{j}$ of type $(2, j)$ and a matrix $D_{j}$ of type $(j, 2)(j \geq 1)$ by

$$
C_{j}:=\left(\begin{array}{llll}
\beta_{2 j+3,1} & \beta_{2 j+3,3} & \ldots & \beta_{2 j+3,2 j-1} \\
\beta_{2 j+5,1} & \beta_{2 j+5,3} & \ldots & \beta_{2 j+5,2 j-1}
\end{array}\right)
$$

and

$$
D_{j}:=\left(\begin{array}{cc}
0 & 0 \\
\vdots & \vdots \\
0 & 0 \\
\beta_{2 j+1,2 j+1} & 0
\end{array}\right) .
$$

Furthermore, we define matrices $A_{j}$ and $\hat{A}_{j}(j \geq 1)$ by

$$
\begin{aligned}
& A_{1}:=\left(\beta_{3,1}\right), \quad A_{2}:=\left(\begin{array}{ll}
\beta_{3,1} & \beta_{3,3} \\
\beta_{5,1} & \beta_{5,3}
\end{array}\right), \\
& A_{j}:=\left(\begin{array}{ll}
A_{j-2} & D_{j-2} \\
C_{j-2} & \left(\begin{array}{ll}
\beta_{2 j-1,2 j-3} & \beta_{2 j-1,2 j-1} \\
\beta_{2 j+1,2 j-3} & \beta_{2 j+1,2 j-1}
\end{array}\right)
\end{array}\right) \quad(j \geq 3)
\end{aligned}
$$

and

$$
\begin{aligned}
& \hat{A}_{1}:=\left(\hat{\beta}_{3,1}\right), \quad \hat{A}_{2}:=\left(\begin{array}{ll}
\beta_{3,1} & \beta_{3,3} \\
\beta_{5,1} & \beta_{5,3}
\end{array}\right), \\
& \hat{A}_{j}:=\left(\begin{array}{ll}
A_{j-2} & D_{j-2} \\
C_{j-2} & \left(\begin{array}{ll}
\beta_{2 j-1,2 j-3} & \beta_{2 j-1,2 j-1} \\
\beta_{2 j+1,2 j-3} & \beta_{2 j+1,2 j-1}
\end{array}\right)
\end{array}\right)(j \geq 3) .
\end{aligned}
$$


From the definition of $P_{2 j+1,1}\left(\lambda_{1}, \cdots, \lambda_{2 j}\right)$, we have

$$
\begin{aligned}
P_{2 j+1,1}\left(\lambda_{1}, \cdots, \lambda_{2 j}\right) & =(-1)^{j-1}\left|A_{j}\right| \\
& =(-1)^{j-1} \mid \begin{array}{cc}
A_{j-2} & D_{j-2} \\
C_{j-2} & \left(\begin{array}{ll}
\beta_{2 j-1,2 j-3} & \beta_{2 j-1,2 j-1} \\
\beta_{2 j+1,2 j-3} & \beta_{2 j+1,2 j-1}
\end{array}\right)
\end{array} \text {. }
\end{aligned}
$$

Substituting $\left(b_{2 j+1,2 j-1}\right)$ to this equality and using the linearity of the determinant for the final column, we have

$$
\begin{aligned}
& P_{2 j+1,1}\left(\lambda_{1}, \cdots, \lambda_{2 j}\right)
\end{aligned}
$$

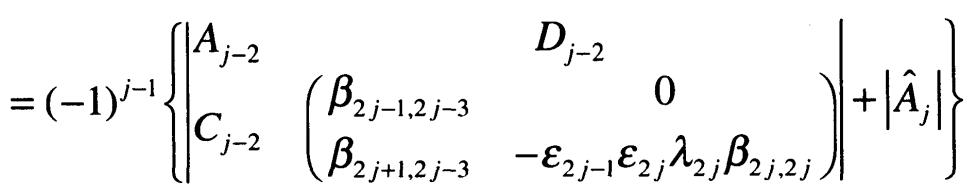

$$
\begin{aligned}
& =(-1)^{j-1}\left\{-\varepsilon_{2 j-1} \varepsilon_{2 j} \lambda_{2 j} \beta_{2 j, 2 j}\left|A_{j-1}\right|+\left|\hat{A}_{j}\right|\right\} \\
& =\varepsilon_{2 j-1} \varepsilon_{2 j} \lambda_{2 j} \beta_{2 j, 2 j} P_{2 j-1,1}\left(\lambda_{1}, \cdots, \lambda_{2 j-2}\right)+(-1)^{j-1}\left|\hat{A}_{j}\right| \quad(j \geq 2) \text {. }
\end{aligned}
$$

Next we shall show $\left|\hat{A}_{j}\right|=0(j \geq 1)$. Clearly we have $\left|\hat{A}_{j}\right|=\left|\hat{\beta}_{3,1}\right|=0$. Assume that $\left|\hat{A}_{j}\right|=0$ for every $j \leq k$. Substituting $\left(b_{2 k+1,2 k-1}\right),\left(b_{2 k+3,2 k-1}\right), \beta_{2 k+1,2 k+1}=\lambda_{2 k+1} \beta_{2 k, 2 k}$ and $\hat{\beta}_{2 k+3,2 k+1}=\lambda_{2 k+1} \beta_{2 k+2,2 k}$ to

$$
\left|\hat{A}_{k+1}\right|=\left|\begin{array}{ll}
A_{k-1} & D_{k-1} \\
C_{k-1} & \left(\begin{array}{ll}
\beta_{2 k+1,2 k-1} & \beta_{2 k+1,2 k+1} \\
\beta_{2 k+3,2 k-1} & \beta_{2 k+3,2 k+1}
\end{array}\right)
\end{array}\right|
$$

and adding $\frac{\varepsilon_{2 k-1} \varepsilon_{2 k} \lambda_{2 k}}{\lambda_{2 k+1}}$ multiple of the final column to the $k$-th column, we obtain

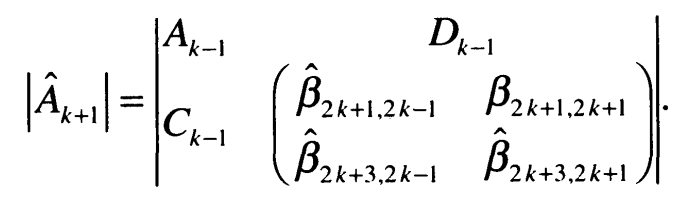

Expanding this determinant with respect to the final column and using the assumption of the induction, we obtain

$$
\begin{aligned}
\left|\hat{A}_{k+1}\right|=-\beta_{2 k+1,2 k+1} & \left|\left(\begin{array}{lll}
\beta_{2 k-1,1} & \cdots & \beta_{2 k-1,2 k-5} \\
\beta_{2 k+3,1} & \cdots & \beta_{2 k+3,2 k-5}
\end{array}\right)\left(\begin{array}{ll}
\beta_{2 k-1,2 k-3} & \beta_{2 k-1,2 k-1} \\
\beta_{2 k+3,2 k-3} & \hat{\beta}_{2 k+3,2 k-1}
\end{array}\right)\right| \\
+ & \hat{\beta}_{2 k+3,2 k+1}\left|\hat{A}_{k}\right|
\end{aligned}
$$




$$
=-\beta_{2 k+1,2 k+1}\left|\left(\begin{array}{lll}
\beta_{2 k-1,1} & \cdots & \beta_{2 k-1,2 k-5} \\
\beta_{2 k+3,1} & \cdots & \beta_{2 k+3,2 k-5}
\end{array}\right)\left(\begin{array}{ll}
\beta_{2 k-1,2 k-3} & \beta_{2 k-1,2 k-1} \\
\beta_{2 k+3,2 k-3} & \hat{\beta}_{2 k+3,2 k-1}
\end{array}\right)\right|
$$

By repeating the same process, we can obtain

$$
\begin{aligned}
\left|\hat{A}_{k+1}\right| & \left.=(-1)^{k-2} \beta_{7,7} \beta_{9,9} \cdots \beta_{2 k+1,2 k+1} \mid \begin{array}{c}
A_{1} \\
\beta_{5,1} \\
\beta_{2 k+3,1}
\end{array}\right)\left(\begin{array}{ccc}
\beta_{5,3} & D_{1} \\
\beta_{2 k+3,3} & \hat{\beta}_{2 k+3,5}
\end{array}\right) \mid \\
& =(-1)^{k-2} \beta_{7,7} \beta_{9,9} \cdots \beta_{2 k+1,2 k+1}\left|\begin{array}{ccc}
\beta_{3,1} & \beta_{3,3} & 0 \\
\beta_{5,1} & \beta_{5,3} & \beta_{5,5} \\
\beta_{2 k+3,1} & \beta_{2 k+3,3} & \hat{\beta}_{2 k+3,5}
\end{array}\right| \\
& =(-1)^{k-1} \beta_{5,5} \beta_{7,7} \cdots \beta_{2 k+1,2 k+1}\left|\begin{array}{cc}
\beta_{3,1} & \beta_{3,3} \\
\beta_{2 k+3,1} & \hat{\beta}_{2 k+3,3}
\end{array}\right| \\
& =(-1)^{k} \beta_{3,3} \beta_{5,5} \cdots \beta_{2 k+1,2 k+1}\left|\hat{\beta}_{2 k+3,1}\right| \\
& =0 .
\end{aligned}
$$

Thus, by the induction, we can conclude $\left|\hat{A}_{j}\right|=0$ every $j \geq 1$. Substituting $\left|\hat{A}_{j}\right|=0$ to (2.6), we have

$$
P_{2 j+1,1}\left(\lambda_{1}, \cdots, \lambda_{2 j}\right)=\varepsilon_{2 j-1} \varepsilon_{2 j} \lambda_{2 j} \beta_{2 j, 2 j} P_{2 j-1,1}\left(\lambda_{1}, \cdots, \lambda_{2 j-2}\right)(j \geq 2) .
$$

After all we can obtain

$$
\begin{aligned}
& P_{2 i+1,1}\left(\lambda_{1}, \cdots, \lambda_{2 i}\right) \\
& =\varepsilon_{3} \varepsilon_{4} \cdots \varepsilon_{2 i} \lambda_{4} \lambda_{6} \cdots \lambda_{2 i} \beta_{4,4} \beta_{6,6} \cdots \beta_{2 i, 2 i} P_{3,1}\left(\lambda_{1}, \lambda_{2}\right) \\
& =-\varepsilon_{1} \varepsilon_{2} \cdots \varepsilon_{2 i} \lambda_{2} \lambda_{4} \cdots \lambda_{2 i} \beta_{2,2} \beta_{4,4} \cdots \beta_{2 i, 2 i} \\
& =-\varepsilon_{1} \varepsilon_{2} \cdots \varepsilon_{2 i} \lambda_{2} \lambda_{4} \cdots \lambda_{2 i}\left|B_{2 i}\right| .
\end{aligned}
$$

Also, we have the following lemma.

LEMMA 2.5. (i) The normal vector field $\nabla_{v_{0}}^{\perp}{ }^{(2 i)} H(i \geq 1)$ along $\sigma$ is written as

$$
\begin{aligned}
\left(H_{2 i}\right) \quad \nabla_{v_{0}}^{\perp}{ }^{(2 i)} H=\sum_{j=1}^{i-1} Q_{2 i, 2 j-1}\left(\lambda_{1}, \cdots, \lambda_{2 i-2}\right) \nabla_{v_{2 j-1}}^{\perp} H & +\lambda_{1} \lambda_{2} \cdots \lambda_{2 i-1} \nabla_{v_{2 j-1}}^{\perp} H \\
& +N_{2 i}\left(\lambda_{1}, \cdots, \lambda_{2 i-2}\right),
\end{aligned}
$$

where $Q_{2 i, 2 j-1}\left(\lambda_{1}, \cdots, \lambda_{2 i-1}\right)(1 \leq j \leq i-1)$ is a homogeneous polynomial of degree 
$(2 i-1)$ and $N_{2 i}\left(\lambda_{1}, \cdots, \lambda_{2 i-2}\right)$ is a normal vector field-valued polynomial of degree at most $(2 i-2)$,

(ii) The normal vector field $\nabla_{v_{0}}^{\perp{ }^{(2 i+1)}} H(i \geq 1)$ along $\sigma$ is written as

$$
\begin{aligned}
\left(H_{2 i+1}\right) \quad \nabla_{v_{0}}^{\perp}{ }^{(2 i+1)} H=\sum_{j=0}^{i-1} Q_{2 i+1,2 j}\left(\lambda_{1}, \cdots, \lambda_{2 i-1}\right) \nabla_{v_{2 j}}^{\perp} H & +\lambda_{1} \lambda_{2} \cdots \lambda_{2 i} \nabla_{v_{2 j}}^{\perp} H \\
& +N_{2 i+1}\left(\lambda_{1}, \cdots, \lambda_{2 i-1}\right),
\end{aligned}
$$

where $Q_{2 i+1,2 j}\left(\lambda_{1}, \cdots, \lambda_{2 i}\right)(0 \leq j \leq i-1)$ is a homogeneous polynomial of degree $2 i$ and $N_{2 i+1}\left(\lambda_{1}, \cdots, \lambda_{2 i-1}\right)$ is a normal vector field-valued polynomial of degree at most $(2 i-1)$.

Proof. Define a normal bundle-valued $(0, j)$-tensor field $T_{j}$ on $M$ by $T_{1}:=\nabla^{\perp} H \quad$ and $\quad T_{k}\left(X_{1}, \cdots, X_{k}\right):=\left(\bar{\nabla}_{X_{1}} T_{k-1}\right)\left(X_{2}, \cdots, X_{k}\right)(k \geq 2) \quad$ for $\quad X_{1}, \cdots, X_{k} \in T M$, where $\bar{\nabla}$ is the connection induced from $\nabla$ and $\nabla^{\perp}$. We shall show $\left(H_{3}\right)$. By using the definition of $T_{j}$ and the Frenet formula, $\nabla_{v_{0}}^{\perp(3)} H$ is rewritten in terms of $T_{j}$ as follows:

$$
\begin{aligned}
& \nabla_{v_{0}}^{\perp}{ }^{(3)} H=\nabla_{v_{0}}^{\perp}{ }^{(2)}\left(T_{1}\left(v_{0}\right)\right)=\nabla_{v_{0}}^{\perp}\left(T_{2}\left(v_{0}, v_{0}\right)+\lambda_{1} T_{1}\left(v_{1}\right)\right) \\
& =T_{3}\left(v_{0}, v_{0}, v_{0}\right)+\lambda_{1} T_{2}\left(v_{1}, v_{0}\right)+2 \lambda_{1} T_{2}\left(v_{0}, v_{1}\right) \\
& -\varepsilon_{0} \varepsilon_{1} \lambda_{1}{ }^{2} \nabla_{v_{0}}^{\perp} H+\lambda_{1} \lambda_{2} \nabla_{v_{0}}^{\perp} H \\
& =Q_{3,0}\left(\lambda_{1}\right) \nabla_{v_{0}}^{\perp} H+\lambda_{1} \lambda_{2} \nabla_{v_{0}}^{\perp} H+N_{3}\left(\lambda_{1}\right) \text {, }
\end{aligned}
$$

where we set $Q_{3,0}\left(\lambda_{1}\right):=-\varepsilon_{0} \varepsilon_{1} \lambda_{1}{ }^{2}$ and $N_{3}\left(\lambda_{1}\right):=T_{3}\left(v_{0}, v_{0}, v_{0}\right)+\lambda_{1} T_{2}\left(v_{1}, v_{0}\right)+2 \lambda_{1} T_{2}$ $\left(v_{0}, v_{1}\right)$. Thus $\left(H_{3}\right)$ is shown. Similarly, $\left(H_{i}\right)(i \geq 4)$ is also shown.

By using these lemmas, we can prove the following theorem.

THEOREM 2.6. Let $M$ be a totally umbilical pseudo-Riemannian submanifold in $\bar{M}$ isometrically immersed by $f$. Assume that for every proper helix $\sigma$ of order $d$ in $M, \bar{\sigma}(:=f \circ \sigma)$ is a proper helix of order $d$ in $\bar{M}$, where $d$ is a positive integer. Then

(i) if $d$ is odd, then $M$ is totally geodesic,

(ii) if $d$ is even, then $M$ is an extrinsic sphere.

Proof. Assume that $d \geq 3$. Fix $p \in M$. For any orthonormal system $X_{0}, X_{1}, \cdots, X_{d-1}$ of $T_{p} M$ and any positive numbers $\lambda_{1}, \cdots, \lambda_{d-1}$, there exists a proper helix $\sigma$ of order $d$ through $p$ with the curvatures $\lambda_{1}, \cdots, \lambda_{d-1}$ whose Frenet frame field $v_{0}, v_{1}, \cdots, v_{d-1}$ coincide with $X_{0}, X_{1}, \cdots, X_{d-1}$ at $p$. Since $\bar{\sigma}(:=f \circ \sigma)$ is a proper 
helix of order $d$ in $M$, by Lemma 2.3, we have

$$
\nabla_{v_{0}}^{\perp(d-1)} H={ }^{t} \boldsymbol{b}_{d} \boldsymbol{B}_{d-2}{ }^{-1} \boldsymbol{H}_{d-3} .
$$

(i) Let $d=2 i+1$. It follows from 2.4 and Lemma 2.5 that

$$
\begin{aligned}
\nabla_{v_{0}}^{\perp}{ }^{(d-1)} H= & \nabla_{v_{0}}^{\perp}{ }^{(2 i)} H \\
=\sum_{k=1}^{i-1} Q_{2 i, 2 k-1}\left(\lambda_{1}, \cdots, \lambda_{2 i-2}\right) \nabla_{v_{2 k-1}}^{\perp} H & +\lambda_{1} \lambda_{2} \cdots \lambda_{2 i-1} \nabla_{v_{2 i-1}}^{\perp} H \\
& +N_{2 i}\left(\lambda_{1}, \cdots, \lambda_{2 i-2}\right)
\end{aligned}
$$

and

$$
\begin{aligned}
& { }^{t} \boldsymbol{b}_{d} \boldsymbol{B}_{d-2}^{-1} \boldsymbol{H}_{d-3}=\frac{1}{\left|\boldsymbol{B}_{d-2}\right|} \sum_{j=0}^{i-1} P_{d, j+1}\left(\lambda_{1}, \cdots, \lambda_{d-1}\right) \nabla_{v_{0}}^{\perp(2 j)} H \\
& =\frac{1}{\left|\boldsymbol{B}_{d-2}\right|}\left\{P_{d, 1}\left(\lambda_{1}, \cdots, \lambda_{d-1}\right) H+\sum_{j=1}^{i-1} P_{d, j+1}\left(\lambda_{1}, \cdots, \lambda_{d-1}\right)\right. \\
& \left.\left\{\sum_{k=1}^{j-1} Q_{2 j, 2 k-1}\left(\lambda_{1}, \cdots, \lambda_{2 j-2}\right) \nabla_{v_{2 k-1}}^{\perp} H+\lambda_{1} \lambda_{2} \cdots \lambda_{2 j-1} \nabla_{v_{2 j-1}}^{\perp} H+N_{2 j}\left(\lambda_{1}, \cdots, \lambda_{2 j-2}\right)\right\}\right\} .
\end{aligned}
$$

Substituting these equalities to (2.7) and noticing the point $p$, we have

$$
\begin{aligned}
& \left|\boldsymbol{B}_{d-2}\right|\left\{\sum_{k=1}^{i-1} Q_{2 i, 2 k-1}\left(\lambda_{1}, \cdots, \lambda_{2 i-2}\right) \nabla_{X_{2 k-1}}^{\perp} H+\lambda_{1} \lambda_{2} \cdots \lambda_{2 i-1} \nabla_{X_{2 i-1}}^{\perp} H+N_{2 i}\left(\lambda_{1}, \cdots, \lambda_{2 i-2}\right)\right\} \\
= & P_{d, 1}\left(\lambda_{1}, \cdots, \lambda_{d-1}\right) H+\sum_{j=1}^{i-1} P_{d, j+1}\left(\lambda_{1}, \cdots, \lambda_{d-1}\right) \\
& \left\{\sum_{k=1}^{j-1} Q_{2 j, 2 k-1}\left(\lambda_{1}, \cdots, \lambda_{2 j-2}\right) \nabla_{X_{2 k-1}}^{\perp} H+\lambda_{1} \lambda_{2} \cdots \lambda_{2 j-1} \nabla_{X_{2 j-1}}^{\perp} H+N_{2 j}\left(\lambda_{1}, \cdots, \lambda_{2 j-2}\right)\right\}
\end{aligned}
$$

Since the degrees of $\left|\boldsymbol{B}_{d-2}\right|, Q_{2 j, 2 k-1}\left(\lambda_{1}, \cdots, \lambda_{2 j-1}\right)(j>k \geq 1)$ and $P_{d, j}\left(\lambda_{1}, \cdots, \lambda_{d-1}\right)(j \geq 1)$ are $i^{2},(2 j-1)$, and $\left(i^{2}+2 i-2 j+2\right)$, respectively, the left-hand side of $(2.8)$ is a polynomial of degree $\left(i^{2}+2 i-1\right)$, the first term $P_{d, 1}\left(\lambda_{1}, \cdots, \lambda_{d-1}\right) H$ of the righthand side is of degree $\left(i^{2}+2 i\right)$ and other terms of the right-hand side are of degree at most $\left(i^{2}+2 i-1\right)$. Hence, since (2.8) holds for every positive numbers $\lambda_{1}, \cdots, \lambda_{2 i-1}$, we obtain $P_{d, 1}\left(\lambda_{1}, \cdots, \lambda_{d-1}\right) H=0$. From Lemma 2.4-(ii), $P_{d, 1}\left(\lambda_{1}, \cdots\right.$, $\left.\lambda_{d-1}\right) \neq 0$ holds. Therefore, we see that $H=0$ at $p$. By the arbitrarity of $p \in M$, we see that $H \equiv 0$, that is, $M$ is totally geodesic. In case of $d=1$, it is directly deduced from Lemma 2.1 that so is $M$.

(ii) Let $d=2 i$. It follows from (2.5), (2.7) and Lemma 2.5 that 


$$
\begin{aligned}
& \mid \boldsymbol{B}_{d-2}\left\{\sum_{k=0}^{i-2} Q_{2 i-1,2 k}\left(\lambda_{1}, \cdots, \lambda_{2 i-3}\right) \nabla_{X_{2 k}}^{\perp} H+\lambda_{1} \lambda_{2} \cdots \lambda_{2 i-2} \nabla_{X_{2 i-2}}^{\perp} H+N_{2 i-1}\left(\lambda_{1}, \cdots, \lambda_{2 i-3}\right)\right\} \\
(2.9) & =P_{d, 1}\left(\lambda_{1}, \cdots, \lambda_{d-1}\right) \nabla_{X_{0}}^{\perp} H+\sum_{j=1}^{i-2} P_{d, j+1}\left(\lambda_{1}, \cdots, \lambda_{d-1}\right) \\
& \left\{\sum_{k=0}^{j-1} Q_{2 j+1,2 k}\left(\lambda_{1}, \cdots, \lambda_{2 j-1}\right) \nabla_{X_{2 k}}^{\perp} H+\lambda_{1} \lambda_{2} \cdots \lambda_{2 j} \nabla_{X_{2 j}}^{\perp} H+N_{2 j+1}\left(\lambda_{1}, \cdots, \lambda_{2 j-1}\right)\right\} .
\end{aligned}
$$

Since the degrees of $\left|\boldsymbol{B}_{d-2}\right|, Q_{2 j+1,2 k-1}\left(\lambda_{1}, \cdots, \lambda_{2 j-1}\right)(j>k \geq 0)$ and $P_{d, j}\left(\lambda_{1}, \cdots, \lambda_{d-1}\right)$ $(j \geq 1)$ are $\left(i^{2}-i\right), 2 j$ and $\left(i^{2}+i-2 j\right)$, respectively, both sides of (2.9) are polynomials of degree $\left(i^{2}+i-2\right)$. Hence, since (2.9) holds for every positive numbers $\lambda_{1}, \cdots, \lambda_{2 i-2}$, terms of degree $\left(i^{2}+i-2\right)$ of the both sides are mutually equal, that is,

$$
\begin{aligned}
& \left|\boldsymbol{B}_{d-2}\right|\left\{\sum_{k=0}^{i-2} Q_{2 i-1,2 k}\left(\lambda_{1}, \cdots, \lambda_{2 i-3}\right) \nabla_{X_{2 k}}^{\perp} H+\lambda_{1} \lambda_{2} \cdots \lambda_{2 i-2} \nabla_{X_{2 i-2}}^{\perp} H\right\} \\
& =P_{d, 1}\left(\lambda_{1}, \cdots, \lambda_{d-1}\right) \nabla_{X_{0}}^{\perp} H+\sum_{j=1}^{i-2} P_{d, j+1}\left(\lambda_{1}, \cdots, \lambda_{d-1}\right) \\
& \left\{\sum_{k=0}^{j-1} Q_{2 j+1,2 k}\left(\lambda_{1}, \cdots, \lambda_{2 j-1}\right) \nabla_{X_{2 k}}^{\perp} H+\lambda_{1} \lambda_{2} \cdots \lambda_{2 j} \nabla_{X_{2 j}}^{\perp} H\right\} .
\end{aligned}
$$

Furthermore, since this equality holds for every orthonormal system $X_{0}, X_{2}, \cdots, X_{2 i-2}$ of $T_{p} M$, we see that $\left|\boldsymbol{B}_{d-2}\right| \lambda_{1} \lambda_{2} \cdots \lambda_{2 i-2} \nabla_{X_{2 i-2}}^{\perp} H=0$, that is $\nabla_{X_{2 i-2}}^{\perp} H=0$. By the arbitrarity of $X_{2 i-2}$, we see that $\nabla^{\perp} H=0$ at $p$. Furthermore, from the arbitrarily of $p \in M, \nabla^{\perp} H \equiv 0$ is deduced. Thus $M$ is an extrinsic sphere. In case of $d=2$, it is directly deduced from Lemma 2.1 that so is $M$.

In the case where $M$ and $\bar{M}$ are Riemannian manifolds, this theorem is written as follows.

COROLLARY 2.7. Let $M$ be a totally umbilical submanifold in a Riemannian manifold $\bar{M}$ isometrically immersed by $f$. Assume that for every helix $\sigma$ of order d in $M, \bar{\sigma}(:=f \circ \sigma)$ is a helix of order $d$ in $\bar{M}$, where $d$ is a positive integer. Then

(i) if $d$ is odd, then $M$ is totally geodesic,

(ii) if $d$ is even, then $M$ is an extrinsic sphere.

Also, we can prove the following theorem.

THEOREM 2.8. Let $M$ be a totally umbilical pseudo-Riemannian submanifold in $M$ isometrically immersed by $f$. Assume that for every proper helix $\sigma$ of order $d$ in $M, \bar{\sigma}(:=f \circ \sigma)$ is a proper helix of order $d+1$ in $\bar{M}$, where $d$ is a positive 
integer. Then $d$ is odd and $M$ is an extrinsic sphere.

Proof. Assume that $d \geq 2$. Fix $p \in M$. For any orthonormal system $X_{0}, X_{1}, \cdots, X_{d-1}$ of $T_{p} M$ and any positive numbers $\lambda_{1}, \cdots, \lambda_{d-1}$, there exists a proper helix $\sigma$ of order $d$ through $p$ with the curvatures $\lambda_{1}, \cdots, \lambda_{d-1}$ whose Frenet frame field $v_{0}, v_{1}, \cdots v_{d-1}$ coincide with $X_{0}, X_{1}, \cdots, X_{d-1}$ at $p$. Since $\bar{\sigma}(:=f \circ \sigma)$ is a proper helix of order $d+1$ in $M$, by Lemma 2.3, we have

$$
\nabla_{\boldsymbol{v}_{0}}^{\perp(d)} H={ }^{\prime} \boldsymbol{b}_{d+1} \boldsymbol{B}_{d-1}^{-1} \boldsymbol{H}_{d-2} \text {. }
$$

Suppose that $d$ is even. Let $d=2 i$. It follows from (2.4), (2.10) and Lemma 2.5 that

$$
\begin{aligned}
& \left|\boldsymbol{B}_{d-1}\right|\left\{\sum_{k=1}^{i-1} Q_{2 i, 2 k-1}\left(\lambda_{1}, \cdots, \lambda_{2 i-2}\right) \nabla_{X_{2 k-1}}^{\perp} H+\lambda_{1} \lambda_{2} \cdots \lambda_{2 i-1} \nabla_{X_{2 i-1}}^{\perp} H+N_{2 i}\left(\lambda_{1}, \cdots, \lambda_{2 i-2}\right)\right\} \\
& =P_{d+1,1}\left(\lambda_{1}, \cdots, \lambda_{d}\right) H+\sum_{j=1}^{i-1} P_{d+1, j+1}\left(\lambda_{1}, \cdots, \lambda_{d}\right) \\
& \left\{\sum_{k=1}^{j-1} Q_{2 j, 2 k-1}\left(\lambda_{1}, \cdots, \lambda_{2 j-2}\right) \nabla_{X_{2 k-1}}^{\perp} H+\lambda_{1} \lambda_{2} \cdots \lambda_{2 j-1} \nabla_{X_{2 j-1}}^{\perp} H+N_{2 j}\left(\lambda_{1}, \cdots, \lambda_{2 j-2}\right)\right\} .
\end{aligned}
$$

Since (2.11) holds for every positive numbers $\lambda_{1}, \cdots, \lambda_{2 i-1}$, by noticing the term of the highest degree, we have $P_{d+1,1}\left(\lambda_{1}, \cdots, \lambda_{d}\right) H=0$. From Lemma 2.4-(ii), $P_{d+1,1}\left(\lambda_{1}, \cdots, \lambda_{d}\right) \neq 0$ holds. Therefore, we obtain $H=0$ at $p$. By the arbitrarity of $p \in M$, we see that $H \equiv 0$, that is, $M$ is totally geodesic. This implies $\bar{d}=d$. Thus a contradiction results. Therefore, $d$ is odd. Let $d=2 i+1$. It follows from (2.5), (2.10) and Lemma 2.5 that

$$
\begin{aligned}
& \left|\boldsymbol{B}_{d-1}\right|\left\{\sum_{k=0}^{i-1} Q_{2 i+1,2 k}\left(\lambda_{1}, \cdots, \lambda_{2 i-1}\right) \nabla_{X_{2 k}}^{\perp} H+\lambda_{1} \lambda_{2} \cdots \lambda_{2 i} \nabla_{X_{2 i}}^{\perp} H+N_{2 i+1}\left(\lambda_{1}, \cdots, \lambda_{2 i-1}\right)\right\} \\
\text { (2.12) } & =P_{d+1,1}\left(\lambda_{1}, \cdots, \lambda_{d}\right) \nabla_{X_{0}}^{\perp} H+\sum_{j=1}^{i-1} P_{d+1, j+1}\left(\lambda_{1}, \cdots, \lambda_{d}\right) \\
& \left\{\sum_{k=0}^{j-1} Q_{2 j+1.2 k}\left(\lambda_{1}, \cdots, \lambda_{2 j-1}\right) \nabla_{X_{2 k}}^{\perp} H+\lambda_{1} \lambda_{2} \cdots \lambda_{2 j} \nabla_{X_{2 j}}^{\perp} H+N_{2 j+1}\left(\lambda_{1}, \cdots, \lambda_{2 j-1}\right)\right\} .
\end{aligned}
$$

Since (2.12) holds for every positive numbers $\lambda_{1}, \cdots, \lambda_{2 i-1}$, by noticing terms of the highest degree, we have

$$
\begin{aligned}
& \left|\boldsymbol{B}_{d-1}\right|\left\{\sum_{k=0}^{i-1} Q_{2 i+1,2 k}\left(\lambda_{1}, \cdots, \lambda_{2 i-1}\right) \nabla_{X_{2 k}}^{\perp} H+\lambda_{1} \lambda_{2} \cdots \lambda_{2 i} \nabla_{X_{2 i}}^{\perp} H\right\} \\
& =P_{d+1,1}\left(\lambda_{1}, \cdots, \lambda_{d}\right) \nabla_{X_{0}}^{\perp} H+\sum_{j=1}^{i-1} P_{d+1, j+1}\left(\lambda_{1}, \cdots, \lambda_{d}\right) \\
& \left\{\sum_{k=0}^{j-1} Q_{2 j+1,2 k}\left(\lambda_{1}, \cdots, \lambda_{2 j-1}\right) \nabla_{X_{2 k}}^{\perp} H+\lambda_{1} \lambda_{2} \cdots \lambda_{2 j} \nabla_{X_{2 j}}^{\perp} H\right\} .
\end{aligned}
$$


Furthermore, since this equality holds for every orthonormal system $X_{0}, X_{2}, \ldots, X_{2 i}$ of $T_{p} M$, we see that $\left|\boldsymbol{B}_{d-1}\right| \lambda_{1} \lambda_{2} \cdots \lambda_{2 i} \nabla_{X_{2 i}}^{\perp} H=0$, that is, $\nabla_{X_{2 i}}^{\perp} H=0$. By the arbitrarity of $X_{2 i}$, we see that $\nabla^{\perp} H=0$ at $p$. Furthermore, from the arbitrarity of $p \in M, \nabla^{\perp} H \equiv 0$ is deduced. Thus $M$ is an extrinsic sphere. In case of $d=1$, it is directly deduced from Lemma 2.1 that so is $M$.

In the case where $M$ and $\bar{M}$ are Riemannian manifolds, this theorem is written as follows.

COROLlARY 2.9. Let $M$ be a totally umbilical submanifold in a Riemannian manifold $M$ isometrically immersed by $f$. Assume that for every helix $\sigma$ of order $d$ in $M, \bar{\sigma}(:=f \circ \sigma)$ is a helix of order $d+1$ in $\bar{M}$, where $d$ is a positive integer. Then $d$ is odd and $M$ is an extrinsic sphere.

\section{§3. Proper helices in an extrinsic sphere.}

Let $M$ be an extrinsic sphere in a pseudo-Riemannian manifold $\bar{M}$ isometrically immersed by $f$ and $\sigma$ a proper helix of order $d$ in $M$. We put $\bar{\sigma}:=f \circ \sigma$. Assume that $\bar{\sigma}$ is a proper curve of order $\bar{d}$. Let $\lambda_{1}, \cdots, \lambda_{d-1}\left(\right.$ resp. $\left.\lambda_{1}, \cdots, \lambda_{\bar{d}_{-1}}\right)$ be the curvatures of $\sigma($ resp. $\bar{\sigma}), v_{0}, \cdots, v_{d-1}$ (resp. $\bar{v}_{0}$, $\left.\ldots, \bar{v}_{\bar{d}_{-1}}\right)$ the Frenet frame field of $\sigma($ resp. $\bar{\sigma})$. For convenience, let $\lambda_{i}=0, v_{i}=0$, $\bar{\lambda}_{j}=0$ and $\bar{v}_{j}=0(i \geq d, j \geq \bar{d})$. Set $\varepsilon_{i}:=g\left(v_{i}, v_{i}\right)$ and $\bar{\varepsilon}_{i}:=\bar{g}\left(\bar{v}_{i}, \bar{v}_{i}\right)(i \geq 0)$. Also, we define $\beta_{i, j}$ and $\beta_{i, j}(i \geq j \geq 1, i+j$ : even $)$ as $(2.1)$.

LEMMA 3.1. The curve $\bar{\sigma}$ is a proper helix in $\bar{M}$ and the vector fields $v_{i}(i \geq 0)$ and $\bar{v}_{j}(j \geq 0)$ along $\sigma$ are related as follows:

$\left(F_{0}^{\prime}\right) \quad \bar{v}_{0}=v_{0}$,

$\left(F_{1}^{\prime}\right) \quad \bar{\beta}_{1}, \bar{v}_{1}=\beta_{1,1} v_{1}+\varepsilon_{0} H$,

$\left(F_{2 i-1}^{\prime}\right) \quad \sum_{j=1}^{i} \bar{\beta}_{2 i-1,2 j-1} \bar{v}_{2 j-1}=\sum_{j=1}^{i} \beta_{2 i-1,2 j-1} v_{2 j-1} \quad(i \geq 2)$,

$\left(F_{2 i}^{\prime}\right) \quad \sum_{j=1}^{i} \bar{\beta}_{2 i, 2 j} \bar{v}_{2 j}=\sum_{j=1}^{i} \beta_{2 i, 2 j} v_{2 j} \quad(i \geq 1)$.

Proof. From (1.1), the Frenet formulas and the assumption that $M$ is totally umbilic, we get

$$
\bar{\lambda}_{1} \bar{v}_{1}=\bar{\nabla}_{v_{0}} v_{0}=\nabla_{v_{0}} v_{0}+\varepsilon_{0} H=\lambda_{1} v_{1}+\varepsilon_{0} H
$$

Thus we obtain $\left(F_{1}^{\prime}\right)$. Furthermore, from this equality, we get 


$$
\bar{\varepsilon}_{1} \bar{\lambda}_{1}^{2}=\varepsilon_{1} \lambda_{1}^{2}+\bar{g}(H, H) .
$$

Since $M$ is an extrinsic sphere, $\bar{g}(H, H)$ is constant. Therefore, $\bar{\lambda}_{1}$ is constant. Operating $\bar{\nabla}_{v_{0}}$ to $\left(F_{1}\right)$, we get

$$
\bar{\beta}_{1,1}\left(-\varepsilon_{0} \bar{\varepsilon}_{1} \bar{\lambda}_{1} v_{0}+\bar{\lambda}_{2} \bar{v}_{2}\right)=\beta_{1,1}\left(-\varepsilon_{0} \varepsilon_{1} \lambda_{1} v_{0}+\lambda_{2} v_{2}\right)-\varepsilon_{0} \bar{g}(H, H) v_{0},
$$

where we use (1.1), (1.2), the Frenet formulas and the assumption that $M$ is an extrinsic sphere. By noticing $\operatorname{Span}\left\{v_{0}\right\}^{\perp}$-component of this equality, we see that

$$
\bar{\lambda}_{2} \bar{\beta}_{1,1} \bar{v}_{2}=\lambda_{2} \beta_{1,1} v_{2}
$$

which implies $\left(F_{2}^{\prime}\right)$ by $(2.1)$. Furthermore, from this equality, we get

$$
\bar{\varepsilon}_{2} \bar{\lambda}_{2}^{2} \bar{\beta}_{1,1}^{2}=\varepsilon_{2} \lambda_{2}^{2} \beta_{1,1}^{2}
$$

which implies that $\bar{\lambda}_{2}$ is constant. Assume that $\left(F_{2 k}^{\prime}\right)$ holds and $\bar{\lambda}_{i}(1 \leq i \leq 2 k)$ are constant. Since $\beta_{2 k, 2 j}\left(\right.$ resp. $\left.\bar{\beta}_{2 k, 2 j}\right)(1 \leq i \leq k)$ are polynomials with variables $\lambda_{1}, \cdots, \lambda_{2 k}$ (resp. $\bar{\lambda}_{1}, \cdots, \bar{\lambda}_{2 k}$ ), these are constant along $\sigma$. Hence, operating $\bar{\nabla}_{v_{0}}$ to $\left(F_{2 k}^{\prime}\right)$, we have

$$
\sum_{j=1}^{k} \bar{\beta}_{2 k, 2 j} \bar{\nabla}_{v_{0}} \bar{v}_{2 j}=\sum_{j=1}^{k} \beta_{2 k, 2 j} \nabla_{v_{0}} v_{2 j}
$$

where we use (1.1) and the assumption that $M$ is an extrinsic sphere. Applying the Frenet formulas and $(2.1)$ to this equality, we obtain $\left(F_{2 k+1}^{\prime}\right)$. Furthermore, from $\left(F_{2 k+1}^{\prime}\right)$, we get

$$
\sum_{j=1}^{k+1} \bar{\varepsilon}_{2 j-1} \bar{\beta}_{2 k+1,2 j-1}^{2}=\sum_{j=1}^{k+1} \varepsilon_{2 j-1} \beta_{2 k+1,2 j-1}^{2}
$$

that is,

$$
\bar{\varepsilon}_{2 k+1} \bar{\beta}_{2 k+1,2 k+1}^{2}=\sum_{j=1}^{k+1} \varepsilon_{2 j-1} \beta_{2 k+1,2 j-1}^{2}-\sum_{j=1}^{k} \bar{\varepsilon}_{2 j-1} \bar{\beta}_{2 k+1,2 j-1}^{2} .
$$

Since $\beta_{2 k+1,2 j-1}(1 \leq j \leq k+1)$ are polynomials with variables $\lambda_{1}, \cdots, \lambda_{2 k+1}$ and $\bar{\beta}_{2 k+1,2 j-1}(1 \leq i \leq k)$ are polynomials with variables $\bar{\lambda}_{1}, \cdots, \bar{\lambda}_{2 k}$, these are constant along $\sigma$, that is, the right-hand side of (3.1) is constant along $\sigma$. Also, the lefthand side of (3.1) is equal to $\bar{\varepsilon}_{2 k+1} \bar{\lambda}_{1}^{2} \bar{\lambda}_{2}^{2} \cdots \bar{\lambda}_{2 k+1}^{2}$. Therefore, we see that $\bar{\lambda}_{2 k+1}$ is constant. Since $\beta_{2 k+1,2 j-1}$ (resp. $\left.\bar{\beta}_{2 k+1,2 j-1}\right)(1 \leq j \leq k+1)$ are polynomials with variables $\lambda_{1}, \cdots, \lambda_{2 k+1}$ (resp. $\left.\bar{\lambda}_{1}, \cdots, \bar{\lambda}_{2 k+1}\right)$, these are constant along $\sigma$. Hence, operating $\bar{\nabla}_{v_{0}}$ to $\left(F_{2 k+1}^{\prime}\right)$, we have 


$$
\sum_{j=1}^{k+1} \bar{\beta}_{2 k+1,2 j-1} \bar{\nabla}_{v_{0}} \bar{v}_{2 j-1}=\sum_{j=1}^{k+1} \beta_{2 k+1,2 j-1} \nabla_{v_{0}} v_{2 j-1}
$$

where we use (1.1) and the assumption that $M$ is an extrinsic sphere. Applying the Frenet formulas and $(2.1)$ to this equality, we obtain $\left(F_{2 k+2}^{\prime}\right)$. Furthermore, from $\left(F_{2 k+2}^{\prime}\right)$, we get

$$
\sum_{j=1}^{k+1} \bar{\varepsilon}_{2 j} \bar{\beta}_{2 k+2,2 j}^{2}=\sum_{j=1}^{k+1} \varepsilon_{2 j} \beta_{2 k+2,2 j}^{2},
$$

that is,

$$
\bar{\varepsilon}_{2 k+2} \bar{\beta}_{2 k+2,2 k+2}^{2}=\sum_{j=1}^{k+1} \varepsilon_{2 j} \beta_{2 k+2,2 j}^{2}-\sum_{j=1}^{k} \bar{\varepsilon}_{2 j} \bar{\beta}_{2 k+2,2 j}^{2}
$$

Since $\beta_{2 k+2,2 j}(1 \leq j \leq k+1)$ are polynomials with variables $\lambda_{1}, \cdots, \lambda_{2 k+2}$ and $\bar{\beta}_{2 k+2,2 j}(1 \leq i \leq k)$ are polynomials with variables $\bar{\lambda}_{1}, \cdots, \bar{\lambda}_{2 k+1}$, these are constant along $\sigma$, that is, the right-hand side of (3.2) is constant along $\sigma$. Also, the lefthand side of $(3.2)$ is equal to $\bar{\varepsilon}_{2 k+2} \bar{\lambda}_{1}^{2} \bar{\lambda}_{2}^{2} \ldots \bar{\lambda}_{2 k+2}^{2}$. Therefore, we see that $\bar{\lambda}_{2 k+2}$ is constant. Thus, by the induction, we see that $\left(F_{i}^{\prime}\right)(i \geq 0)$ hold and $\lambda_{j}(j \geq 1)$ are constant (i.e., $\bar{\sigma}$ is a proper helix).

By using this lemma, we can prove the following theorem.

THEOREM 3.2. Let $M$ be an extrinsic sphere in a pseudo-Riemannian manifold $M$ isometrically immersed by $f$ and $\sigma$ a proper helix of order $d$ in $M$ such that $\bar{\sigma}(:=f \circ \sigma)$ is a proper curve in $\bar{M}$, where $d$ is a positive integer. Then

(i) if $d$ is odd, then $\bar{\sigma}$ is a proper helix of order $d$ or $d+1$,

(ii) if $d$ is even, then $\bar{\sigma}$ is a proper helix of order $d$.

Proof. Let $v_{i}(0 \leq i \leq d-1)\left(\right.$ resp. $\left.v_{i}(0 \leq i \leq \bar{d}-1)\right)$ the Frenet frame field of $\sigma($ resp. $\bar{\sigma})$ and, for convenience, $v_{i}=0(i \geq d)$ and $\bar{v}_{i}=0(i \geq \bar{d})$. According to Lemma 3.1, $\bar{\sigma}$ is a proper helix, $\bar{v}_{2 i} \in \operatorname{Span}\left\{v_{0}, v_{2}, \cdots, v_{2 i}\right\}(i \geq 0)$ and $\bar{v}_{2 i+1} \in \operatorname{Span}\left\{v_{1}, v_{3}, \cdots, v_{2 i+1}, H\right\}(i \geq 0)$. The conclusion is directly deduced from these facts.

In the case where $M$ and $\bar{M}$ are Riemannian manifolds, this theorem is written as follows.

COROLlaRY 3.3. Let $M$ be an extrinsic sphere in a Riemannian manifold $\bar{M}$ isometrically immersed by fand $\sigma$ a helix of order $d$ in $M$, where $d$ is a positive integer. Then 
(i) if $d$ is odd, then $f \circ \sigma$ is a helix of order $d$ or $d+1$,

(ii) if $d$ is even, then $f \circ \sigma$ is a helix of order $d$.

\title{
References
}

[1] Abe, N. , Nakanishi Y. and Yamaguchi, S., Circles and spheres in pseudo-Riemannian geometry, Aequationes Mathematicae 39 (1990), 134-145.

[2] Nakagawa, H., On a certain minimal immersion of a Riemannian manifold into a sphere, Kôdai Math. J. 3 (1980), 321-340.

[ 3 ] Nakanishi, Y., On curves in pseudo-Riemannian submanifolds, Yokohama Math. J. 36 (1988), 137-146.

[ 4 ] Nakanishi, Y., On helices and pseudo-Riemannian submanifolds, Tsukuba J. of Math. 12 (1988), 469-476.

[ 5 ] Nomizu K. and Yano, K., On circles and spheres in Riemannian geometry, Math. Ann. 210 (1974), 163-170.

[ 6 ] O’Neill, B., Semi-Riemannian geometry, Academic Press, New York, 1983.

[ 7 ] Sakamoto, K. , Planar geodesic immersions, Tôhoku Math. J. 29 (1977), 25-56.

[ 8 ] Sakamoto, K., Helical immersions into a unit sphere, Math. Ann. 261 (1982), 63-80.

\author{
Department of Mathematics \\ Korea University \\ 1-700 Ogawa-cho Kodaira \\ Tokyo 176 \\ Japan, \\ Professional Services \\ Account Consulting \\ AT\&T JAPAN \\ 16-16, Nampeidai-cho Shibuya-ku \\ Tokyo 150 \\ Japan \\ and \\ Department of Mathematics \\ Faculty of Science \\ Science University of Tokyo \\ 26 Wakamiya Shinjuku-ku, \\ Tokyo 162 \\ Japan
}

